\title{
The Role of Telomeres and Telomerase in Cancer Research
}

\author{
Sheila A. Stewart and \\ Department of Cell Biology and Physiology and Medicine, Washington University School of \\ Medicine, 660 South Euclid Avenue, Campus Box 8228, St. Louis, MO 63110, Phone: \\ 314-362-7437, Fax: 314-362-7463
}

\author{
Alison A. Bertuch \\ Baylor College of Medicine, Department of Pediatrics, Hematology/Oncology Section, One Baylor \\ Plaza, Houston, TX 77030, Tel: 832-824-4579, FAX: 832-825-4651
}

Sheila A. Stewart: sheila.stewart@wustl.edu; Alison A. Bertuch: abertuch@txccc.org

\begin{abstract}
The fourth AACR Special Conference on The Role of Telomeres and Telomerase in Cancer Research was held February 27-March 2, 2010 in Fort Worth, Texas. The meeting was organized to bring together those interested in the basic molecular mechanisms that govern telomere dynamics and stability with those interested in the clinical implications of telomere dysfunction and the use of telomeres and telomerase as therapeutic targets. The meeting was extremely successful as evidenced by the attendance and quality of the presentations. Indeed, several important themes emerged including 1) the intricate connection between the DNA replication and repair machineries in basic telomere replication and stability; 2) the complex interplay between the telomere-specific shelterin components and DNA repair proteins; 3 ) the non-telomeric functions of TERT in numerous cell types including stem cells; 4) a growing appreciation for the connection that exists between telomere maintenance deficiency states and diverse conditions such as idiopathic pulmonary fibrosis and hematopoietic malignancies; and 5) the successful progression of agents targeting telomerase directly and immunologically to Phase III clinical trials. Evident at the meeting was the vibrant energy that permeates the telomere field and the important biological and medical findings that it continues to yield.
\end{abstract}

\section{Introduction}

The telomere is a complex DNA-protein structure located at the end of all linear eukaryotic chromosomes. The functional importance of the telomere has been recognized since the 1940's but the mechanisms that maintain this critical structure are still being elucidated today. The cell is exquisitely sensitive to DNA double strand breaks and detection of such breaks leads to a cascade of cellular responses that culminate in DNA repair. Because the natural chromosome terminus could be perceived as a DNA break, it was originally proposed that the telomere "disguised" chromosome ends from the DNA damage surveillance machinery and even excluded DNA repair factors from accessing the chromosome end. While this original hypothesis made some sense, observations over the last decade and a half from a wide range of organisms have underscored the intimate connection telomere stability shares with the activities of the DNA repair machinery. The importance of this connection was highlighted at the recent AACR Special Conference on The Role of Telomeres and Telomerase in Cancer Research meeting that occurred in Fort Worth, Texas, February 27-March 2, 2010. The meeting brought together researchers who focus on telomere biology and/or basic DNA 
replication and repair with those interested in the role of telomere stability (or loss thereof) in disease and how telomeres and telomerase might be targeted in cancer therapeutics. The meeting underscored the challenge those in the telomere field face in delineating the molecular activities the DNA replication and repair machineries play at the telomere and how the telomere complex modifies, modulates and/or dampens the activities of these proteins to ensure chromosome stability. In addition, the meeting highlighted the role the telomere plays in cancer and other conditions including dyskeratosis congenita and idiopathic pulmonary fibrosis, and the various therapeutic approaches that have emerged from our current knowledge of telomere biology. In this short report, we attempt to review some of the key presentations from this meeting and apologize to the numerous researchers whose work we do not have the space to highlight.

\section{A Role for Rap1}

Telomere stability is ensured by the retention of sufficient telomeric DNA reserves and the activities of numerous proteins including the shelterin complex (consisting of TRF1, TRF2, TIN2, TPP1, POT1, and RAP1) and basic DNA replication and repair proteins. While there remains a great deal to learn, we now have a reasonable understanding of the roles TRF1, TRF2, TIN2, TPP1, and POT1 play in mammalian telomere stability. In contrast, the contribution of RAP1 is just now emerging. At this meeting, two groups reported on mice bearing conditional RAP1 alleles, which revealed functions of RAP1 at the mammalian telomere. Titia de Lange (Rockefeller University, New York, NY) reported that conditional loss of RAP1 alone had little impact on telomere stability (i.e. no overt changes in telomere length, telomere DNA damage foci or telomeric fusions were noted) (1). However, when combined with loss of $\mathrm{Ku}$, there was a marked increase in telomere sister chromatid exchanges, suggesting that RAP1 participates in inhibiting homology directed repair at the telomere.

However, as is becoming a theme with telomere "specific" proteins, Paula Martinez from Maria Blasco's group (Spanish National Cancer Centre (CNIO), Madrid, Spain) went on to propose that RAP1 has extra-telomeric functions as well. Using expression array and ChIP-Seq analysis, Martinez demonstrated that RAP1 not only localized to telomeres as expected but was also found at extra-telomeric sites. Interestingly, extra-telomeric RAP1 binding sites are particularly abundant at subtelomeric regions that contain known genes. Analysis of gene expression in RAP1-deficient cells revealed that many of the genes located in the regions bound by RAP-1 were deregulated, suggesting that RAP1 plays a role in subtelomeric gene silencing and/or in transcriptional control (2). These findings argue that mammalian RAP1 functions analogously to its yeast counterpart, which has dual functions at the telomere and in transcriptional regulation. Notably, whereas neither the de Lange nor Blasco groups found a significant role for RAP1 in inhibiting telomere fusions, a recent report by Baumann and colleagues suggests this may be the case in human cells (3). The difference in these findings may be due to the species under investigation (i.e. mouse versus human, which in the case of $\mathrm{Ku}$ deletion results in drastically different phenotypes) or the experimental approaches taken by each group. Indeed the de Lange and Blasco groups used a loss of function approach while the Baumann group utilized ectopic expression of a fusion protein, which could account for the differences in the studies.Nonetheless, these findings raise the interesting possibility that RAP1 has additional functions at the human telomere.

\section{DNA repair factors and telomeres}

Eric Hendrickson (University of Minnesota, Minneapolis, MN) further emphasized the point that, while the mouse is indispensable to our work, we must keep in mind that important differences with human exist. Hendrickson, a self-proclaimed "Ku-ologist", has used recombinant adenovirus-associated virus (AAV) to create a human cancer cell line that expresses a conditional null allele of the large Ku subunit, Ku86 (XRCC5) (4). Whereas the 
loss of $\mathrm{Ku}$ in murine cells results in relatively modest telomere phenotypes, including increased telomere sister chromatid exchanges and equivocal changes in telomere length, Hendrickson's group found that conditional loss of human Ku86 resulted in complete loss of 70-90\% of telomeres by fluorescence in situ hybridization and a significant increase in Lig4-independent sister telomere fusions. They also observed a significant increase in extrachromosomal telomeric repeat-containing circular DNA, so-called T circles, suggesting that Ku plays a major role in suppressing catastrophic homologous recombination (HR) at the human telomere. Taken together with the RAP1 story and previous work, it is clear that both shelterin components and DNA repair proteins coordinately suppress inappropriate HR and nonhomologous end joining (NHEJ) at the telomere.

Researchers focused on basic DNA repair mechanisms including Tanya Paull (University of Texas, Austin, TX), Frederick Alt (Children's Hospital Boston, Boston, MA), and Andre Nussenzweig (National Cancer Institute, Bethesda, MD) presented work highlighting the biochemical activities of the Mre11/Rad50/Xrs2 complex and molecular activities XLF, and BRCA1, respectively, in genomic stability. These presentations reminded the audience that the details that distinguish the activities of these proteins at the telomere from those across the genome are of critical importance to our overall understanding of telomere biology. A presentation from another DNA repair researcher, John Petrini (Memorial Sloan-Kettering Cancer Center, New York, NY) underscored this basic idea. In his presentation, Petrini showed that loss of the MRE11 protein in cells deleted of TRF2 reduced the number of chromosome fusions normally observed upon TRF2 loss (5). This finding was in agreement with previous work demonstrating that upon telomere uncapping, the 3' single strand overhang is processed by the DNA repair machinery before the NHEJ machinery can act (6). Based on the known functions of MRE11, highlighted by Tanya Paull, this finding indicates that the MRE11 complex plays an important role in processing the 3' single strand overhang.

\section{The DNA replication machinery and telomeres}

In addition to the activities of the DNA repair machinery, recent published work and data presented at this meeting also highlighted the critical interplay between the DNA replication machinery and members of the shelterin complex at the telomere. Previous work from the de Lange and Blasco groups demonstrated that TRF1, whose roles were previously thought to be restricted to the negative regulation of telomere length and sister telomere cohesion, is critical for replication fork progression through the telomere $(7,8)$. Indeed, they demonstrated that conditional loss of TRF1 led to inefficient replication fork progression through the telomere and telomere dysfunction characterized by multiple telomere signals referred to by some as fragile telomeres. Fuyuki Ishikawa (Kyoto University, Kyoto, Japan) presented work utilizing the Xenopus system to demonstrate that this central role of TRF1 may be limited to mammals. Indeed, he showed that Xenopus TRF1 doesn't bind the telomere during S phase whereas TRF2 does. He went on to show that depletion of TRF2 led to a $50 \%$ reduction in overall telomeric replication, arguing for a functional importance of TRF2 rather than TRF1 in the frog for telomere replication. Understanding the molecular determinants that lead to these differences will be of great interest.

Several posters and talks further highlighted the importance of basic replication proteins in telomere stability. Building off previous work from the Bohr group, Avik Ghosh (National Institute on Aging, Baltimore, MD) demonstrated that the poorly characterized RECQL4 protein could resolve D loops and stimulate the activities of the WRN protein at telomeric D loops but not non-telomeric sequences in vitro. Given that Jan Karlseder's group (Salk Institute for Biological Sciences) previously found that WRN is important for telomere replication (9), these findings suggest that like WRN, RECQL4 may participate in telomere metabolism. A presentation from Sheila Stewart (Washington University, St. Louis, MO) demonstrated that 
the Okazaki fragment processing protein FEN1 also plays a crucial role in telomere replication. Indeed, FEN1's ability to participate in DNA repair, presumably in the resolution or stabilization of stalled replication forks and not its participation in basic lagging strand replication, was necessary for telomere replication while being dispensable for genome-wide replication. Together, these talks underscored the important role basic DNA replication factors may play in telomere replication and maintenance.

Continuing along the theme of telomere replication, Bradley Stohr from Elizabeth Blackburn's group (University of California, San Francisco, CA) provided potential insight into why telomeres evolved to be G-rich, despite the challenge they pose to replication machinery. He demonstrated that by sequentially changing the terminal telomeric sequences to progressively more self-complementary sequences, cells began to display increased levels of sister telomere fusions, something rarely encountered even in the face of massive telomere dysfunction. This finding leads to the intriguing possibility that in vertebrates, evolution selected for the non-self complementary TTAGGG sequence to minimize the probability of sister telomere fusions but at the cost of difficult to replicate sequences sensitive to loss of function mutations in basic DNA replication proteins.

\section{Timing, coordination and regulation of telomerase}

Although it has been nearly 25 years since the seminal discovery of telomerase by recent Nobel laureates Carol Greider (Johns Hopkins University, Baltimore, MD) and Elizabeth Blackburn (University of California, San Francisco, CA), the molecular details governing telomerase action continue to emerge. A number of the meeting's presentations sought to address some of the key outstanding questions. Virginia Zakian (Princeton University, Princeton, NJ) reported on her group's continued efforts to decipher the series of interactions governing the recruitment of telomerase to telomeres in the Saccharomyces cerevisiae model system. Building on her prior studies that indicated Xrs2 targets Tel1 [the yeast ataxia telangiectasia mutated (ATM) ortholog] to short telomeres, which in turn promotes the preferential association of telomerase to these ends (10), she presented data indicating that Xrs2's association with short telomeres is facilitated by the reduced occupancy of Rif2. Thus, Rif2 may prove to be the first signal in the pathway identifying short telomeres in the budding yeast model system.

Carolyn Price (University of Cincinnati, Cincinnati, $\mathrm{OH}$ ) presented her group's ongoing work in the model system that first yielded telomerase, Tetrahymena thermophila. She demonstrated that Tetrahymena cells express a developmentally regulated Pot 1 paralog, Pot $1 \mathrm{~b}$, which appears to function in the pathway of chromosome breakage and de novo telomere synthesis during macronucleus formation. Interesting in its own regard, this work reminds us that even humans may harbor novel telomere-associated factors or splice variants of known factors that are developmentally regulated and missed in laboratory cell culture systems.

Joachim Lingner (Ecole Polytechnique Fédérale de Lausanne, Lausanne, Switzerland) reported on studies seeking to define the proteins that receive telomerase at mammalian telomeres. Using cells overexpressing telomerase, his group found less TERT and TERC at telomeres in cells deficient for TPP1 or TIN2 but no change in cells deficient for POT1 (11). Because both TPP1 and TIN2 are thought to negatively regulate telomerase, these localization studies may ultimately lead to unexpected insights into telomerase's association and activity at telomeres. Tpp1's role as a regulator of TERT at chromosome ends was also revealed by studies presented by Rosa Marion from Maria Blasco's group. The Blasco group demonstrated previously that telomere elongation during nuclear reprogramming of mouse embryonic fibroblasts into induced pluripotent stem cells (iPS cells) is mediated by telomerase (12). These finding were expanded on at this meeting when Marion showed that Tpp1-deficient MEFs failed to elongate 
telomeres during nuclear reprogramming into iPS cells, demonstrating that Tpp1 is essential for net telomere elongation by telomerase in vivo (13).

Woodring Wright's (UT Southwestern Medical Center, Dallas, TX) talk covered many fundamental questions regarding the action, timing and coordination of telomerase in human cells. Previous studies in the budding yeast model demonstrated that telomerase acts only on the shortest telomeres, after bulk DNA replication (i.e., in late S phase) and coordinately with the lagging strand synthesis machinery, which is required for telomere C-strand fill-in after telomerase-mediated elongation (14). The preferential elongation of the shortest telomeres has also been demonstrated in mouse. Recent work in human cancer cells from his and Jerry Shay's group has revealed a different picture. Utilizing a series of clever assays that allowed for monitoring of G-strand elongation and C-strand fill, they found that, under conditions of telomere length homeostasis, telomerase acts on most, not just the shortest, telomeres in $\mathrm{S}$ phase and that C-strand fill-in is temporally dissociated from telomerase elongation, occurring much later in $\mathrm{S}$ phase (15). Wright presented their continuing studies, which indicate telomerase acts processively when telomere length is at steady state or when telomerase is overexpressed, but distributively when elongating shortened telomeres. Thus, even in human cancer cells, the action of telomerase is influenced in some way by telomere length. Wright proposed that telomere length equilibrium conditions might underlie the indiscriminate action of telomerase at nearly all telomeres in these cancer cell lines. Indeed, a recent study demonstrating the preferential elongation of short telomeres in primary human fibroblast cells following expression of telomerase supports this very proposal (16).

Although the abovementioned work illustrates how budding yeast telomere biology may display differences from human, work on S. cerevisae will no doubt continue to provide offerings to the study of human telomeres. Carolyn Price illustrated this point when she described her findings on human $\mathrm{Ctc1}$, a recently characterized protein that interacts with paralogs of budding yeast Stn1 and Ten1 $(17,18)$. Ctc1, like Cdc13, interacts with components of the lagging strand DNA synthesis machinery. The extent to which Ctc1 is specialized for telomere replication and function is of clear interest to many in the field.

\section{Nontelomeric functions of TERT}

Over the last several years an appreciation for the extra-telomeric functions of TERT has emerged. Previous work demonstrated that immortal TERT-positive versus telomerasenegative cells were more resistant to several chemotherapeutic modalities, that ectopic expression of hTERT increased the tumorigenic potential of ALT tumor cells (19) and that transgenic expression of mTERT in otherwise telomerase-positive mice led to increased tumorigenesis (20-22), and increased hair growth when expressed specifically in the skin (23). In addition, TERT was recently shown to function as an RNA-dependent RNA polymerase and participate in the microRNA pathway (24). Together, these and other studies indicate that TERT possesses extra-telomeric functions. This theme continued at the 2010 AACR telomere meeting. Steven Artandi (Stanford University School of Medicine, Stanford, CA) presented a compelling story demonstrating that TERT interacts directly with WNT transcription factors and cooperates in activating WNT responsive promoters during normal hair growth (25), adding yet another level of complexity to the TERT story. In addition, Falk Mancke (Comprehensive Cancer Center Freiburg, Freiburg, Germany) demonstrated that telomerase interacts with $\beta$-catenin in cells from late stage adenocarcinomas of the esophagus. However, there is no such interaction in early stages of adeno or squamous cancer, i.e. irrespective of their differentiation, arguing that this interaction may be important in tumor progression. Studies such as these have important implications when considering the cellular consequences of TERT upregulation in the vast majority of human cancers. Clearly 
investigation into the extra-telomeric roles of TERT will remain an exciting area that will continue to challenge our understanding of telomerase biology.

\section{Consequences of telomere dysfunction}

First implicated in the bone marrow predisposition syndrome dyskeratosis congenita, constitutional mutations and single nucleotide polymorphisms (SNPs) in telomerase subunits TERT and TERC are now being reported with a number of diseases with nonsyndromic presentations. Two talks at the meeting emphasized this point. First, Peter Lansdorp (University of British Columbia, Vancouver, BC, Canada) reported a 3 to 6 fold higher frequency of the hypomorphic A1062T TERT allele in individuals with acute myelogenous leukemia, chronic lymphocytic lymphoma (CLL), and diffuse large B-cell lymphoma relative to normal controls (26). He and his group hypothesized that other variants in TERT or TERC might be more frequent in these diseases, and, indeed, full length sequencing of TERT in 70 individuals with CLL revealed germline mutations in TERT in over 10\% of patients. Hypomorphic TERT variants could predispose individuals to the development of various tumors by compromising tissue renewal and increasing cell divisions in remaining cells. This mechanism could explain the linkage between SNPs mapping to the TERT locus in cancer that has been observed in several large linkage studies in which the genome of cancer patients was compared to that of normal controls.

Christine Garcia (UT Southwestern Medical Center, Dallas, TX) described her group's analysis of over 100 individuals with either TERT mutations from 21 different families in which the proband presented with idiopathic pulmonary fibrosis (IPF), a condition that is also seen in individuals with dyskeratosis congenita. They found the development of IPF was age dependent and exacerbated by exposures such as smoking. Only a few mutation carriers developed bone marrow failure or myelodysplastic syndrome, however, the incidence of mild anemia, aplastic anemia and myelodysplastic syndrome appeared higher than would be expected for the general population. In addition, they found progressive telomere shortening in three large five-generation pedigrees, raising the possibility that mutation carriers in later generations may exhibit more of the clinical features characteristic of DC. Hopefully, there will be long-term follow up of these families, which will address this unresolved question.

The association of hypomorphic TERT alleles with malignancy lends circumstantial support to the hypothesis that telomere dysfunction resulting from critically short telomeres may contribute to the genomic changes that drive oncogenesis. Further evidence for this came from Duncan Baird (Cardiff University, Cardiff, Wales) who presented data from his group's study of chromosome specific telomere lengths and fusions in early to late stage CLL. They found that mean telomere lengths were variable in early stage CLL but uniformly short in late stage CLL and that telomere fusions increased coincident with telomere shortening, providing the first direct demonstration of fusion between short dysfunctional telomeres during the progression of cancer in humans. Furthermore, sequence analysis revealed deletions and complex rearrangements similar to those observed in mortal fibroblast cultures undergoing 'crisis'.

Titia de Lange also presented her group's recent work demonstrating that a persistent telomere damage signal can induce tetraploidization, a phenomenon associated with early carcinogenesis. Using p53-deficient POT1a/b double knock out mouse embryonic fibrolbasts, she showed that tetraploidization was strongly suppressed by inhibition of ATR-mediated DNA damage signaling (27). She further showed that when telomere end protection was reestablished after tetraploidization, normal cell cycle divisions resumed. These studies provide new insight into how telomere dysfunction can contribute to the generation of cancer genomes. 


\section{Alternative lengthening of telomeres}

A continued interest in the alternative lengthening of telomeres (ALT) pathway(s), which is observed in a small subset of carcinomas, but larger numbers of mesenchymal and neuroepithelial malignancies, was evident at the meeting. Examples of this include a presentation by Qin Yang (Washington University, St. Louis, MO), who extended his group's work implicating the structure-specific endonuclease, Mus81, in the maintenance of telomeres in ALT by demonstrating that Mus81 localization to ALT-associated promyelocytic leukemia bodies (APBs) was dependent on BLM. Finally, Roger Reddel (Children's Medical Research Institute, Westmead, Australia) reported on his group's recent efforts to determine whether ALT-like activity occurs in nontransformed cells. To do this, they analyzed the spread of a telomeric tag, first introduced into murine embryonic stem cells, in the germline and somatic tissues. Whereas the tag displayed a normal germline Mendelian segregation, even after numerous generations, it was copied to the telomeres of other chromosomes in cells from various somatic tissues. They propose that ALT, like telomerase, may be regulated in normal cells and dysregulated in tumorigenesis.

\section{Telomerase takes center stage in cancer therapeutics}

Because telomerase is poorly or only transiently expressed in human somatic cells, yet upregulated in the vast majority of human cancers, and telomere maintenance is crucial for long-term cellular proliferation, telomerase is an attractive target for anti-cancer therapy. In the meeting's keynote address, Jerry Shay (UT Southwestern Medical Center, Dallas, TX) gave an overview of the various approaches that have been undertaken to exploit telomerase in cancer therapeutics and then focused in on the development status of imetelstat (originally known as GRN163L, Geron Corp., Menlo Park, CA), a lipidated 13-mer oligonucleotide N3' P5'-thio-phosphoramidate that has high affinity and binding specificity for the template region of the telomerase RNA subunit (TERC) and is a potent telomerase inhibitor in all major cancer cell types examined in vitro. He reported that a number of Phase I studies in solid and hematological malignancies have been completed establishing a single agent Phase II dose and an interval schedule. In addition, there is also a Phase I/II study underway that is evaluating imetelstat in combination with paclitaxel and bevacizumab in patients with locally recurrent or metastatic breast cancer. Finally, Phase II studies are also evaluating imetelstat in nonsmall cell lung carcinoma (NSCLC), breast cancer, and multiple myeloma.

While the clinical trials are underway, preclinical studies addressing important aspects of imetelstat efficacy and potential resistance continue at the bench. Several groups are investigating whether tumor initiating cells or cancer stem cells might be preferentially targeted by telomerase inhibition. For example, Shay's group demonstrated that telomerase activity in putative tumor initiating cells isolated from primary human glioblastoma multiforme and prostate cancer was inhibited by imetelstat treatment. In addition, they observed telomere shortening following prolonged imetelstat treatment. Imetelstat treated GBM tumor initiating cells also exhibited decreased neurosphere formation and tumor formation in a murine tumor explant model, suggesting that these critical cells may be viable in vivo targets. Similarly, Robert Tressler (Geron, Menlo Park, CA) reported that imetelstat had a profound impact on cancer stem stems derived from melanoma, breast and pancreatic carcinoma, multiple myeloma, low grade glioma and glioblastoma multiforme. Indeed, broad in vitro and in vivo activity was observed, however, the mechanism of inhibition varied with tumor type. These reports support the notion that telomerase inhibition may be particularly effective on these crucial cells in tumor development and survival.

As with all treatments, the potential for the development of resistance to telomerase inhibitors such as imetelstat remains a concern. To address this question, Robin Frink (UTSW Medical 
Center, Dallas, TX) reported results of long-term treatment of NSCLC cell lines with imetelstat. As expected, telomerase inhibition and telomere shortening were uniformly observed initially, however, the effects varied; some cell lines underwent rapid apoptosis, while others showed a decline in proliferation coincident with telomere shortening. The effects of prolonged treatment with imetelstat (in one case over a year) on telomere length varied as well. In one case, telomeres stabilized, whereas in another, telomere length gradually increased to parental length. Whether these effects were due to upregulation of telomerase or secondary mutations/ adaptations is unclear at this point but they underscore the importance of further work into the mechanisms of resistance if telomerase therapies are to be successful.

Telomerase has also been exploited as a tumor associated antigen and potential immunotherapy target. Gary Middleton (St. Luke's Cancer Centre, Guildford, England) highlighted the progress that has been made on telomerase peptide vaccines. Phase I and II studies have been completed with a variety of peptides and demonstrated tolerability, immunological responses, and prolonged survival in responders. GV1001, a synthetic peptide corresponding to hTERT residues 611-626, is currently being evaluated in a Phase III randomized trial comparing gemcitabine and capecitabine with or without GV1001 in patients with locally advanced or metastatic pancreatic cancer and a Phase III study evaluating optimized TERT peptide 572Y (Vx-001) in NSCLC is expected to begin this year. The findings from these studies will be highly anticipated and if positive will firmly establish the feasibility of immunologically targeting TERT in the treatment of human cancers.

\section{Telomere biology and cancer diagnostics}

Other advances that may find their way into the clinic were also presented. For example, Roger Reddel presented his group's recent work on telomeric C-circles, partially single stranded closed circular DNA molecules that contain telomeric repeat tracts (28). Using a sensitive Ccircle assay they developed, greater than 100 fold higher C-circle assay levels were observed in a wide variety of ALT-positive compared to ALT-negative human cell lines. While other markers of ALT have been described, what makes this assay particularly promising is that it also detected higher C-circle assay levels in blood from patients with ALT-positive compared to ALT-negative osteosarcomas, suggesting its potential applicability to diagnosis, treatment and disease surveillance of ALT-positive tumors. Jerry Shay reported that the telomerase repeat amplification protocol (commonly known as the TRAP assay) could be used to detect limiting numbers of telomerase-positive cells in the blood of patients with NSCLC, raising the possibility that it could be tool for early detection/screening modalities. It is possible that other telomere or telomerase-based diagnostic tools will be developed in the years to come.

\section{Summary}

This Special Conference highlighted the fact that, while research on telomeres and telomerase has partially unraveled the complexity governing the maintenance of chromosome ends, there remains a great deal left to better understand such as in vivo telomerase enzymology, functions of integral telomere-specific factors, and the contribution of repair and recombination factors on normal telomere structure and function. At this meeting, we found that the knowledge acquired through basic telomere biology research is being applied to the study of cancer and other disease processes. We anticipate that an understanding of the role telomere maintenance deficiency plays in cancer development and other conditions may lead ultimately to telomerebased risk categories for prevention and surveillance. In addition, it was encouraging to learn that telomerase-targeted therapies are continuing along the drug development pipeline. At the current pace, how some of today's discoveries will impact on our understanding of human disease may be apparent as soon as the next biannual Special Conference on Telomeres and Telomerase in Cancer Research. 


\section{Supplementary Material}

Refer to Web version on PubMed Central for supplementary material.

\section{Acknowledgments}

We thank the meeting organizers, Titia de Lange, Woodring Wright and Virginia Zakian, for putting together an excellent meeting, the AACR for its continued interest in telomere research, and the Series and Award Supporters. We also thank the participants for outstanding oral and poster presentations and engaging discussions.

\section{References}

1. Sfeir A, Kabir S, van Overbeek M, Celli GB, de Lange T. Loss of Rap1 induces telomere recombination in the absence of NHEJ or a DNA damage signal. Science 327:1657-61. [PubMed: 20339076]

2. Martinez P, Thanasoula M, Carlos AR, et al. Mammalian Rap1 controls telomere function and gene expression through binding to telomeric and extratelomeric sites. Nat Cell Biol. In press.

3. Sarthy J, Bae NS, Scrafford J, Baumann P. Human RAP1 inhibits non-homologous end joining at telomeres. EMBO J 2009;28:3390-9. [PubMed: 19763083]

4. Wang Y, Ghosh G, Hendrickson EA. Ku86 represses lethal telomere deletion events in human somatic cells. Proc Natl Acad Sci U S A 2009;106:12430-5. [PubMed: 19581589]

5. Attwooll CL, Akpinar M, Petrini JH. The mre11 complex and the response to dysfunctional telomeres. Mol Cell Biol 2009;29:5540-51. [PubMed: 19667076]

6. Deng Y, Guo X, Ferguson DO, Chang S. Multiple roles for MRE11 at uncapped telomeres. Nature 2009;460:914-8. [PubMed: 19633651]

7. Sfeir A, Kosiyatrakul ST, Hockemeyer D, et al. Mammalian telomeres resemble fragile sites and require TRF1 for efficient replication. Cell 2009;138:90-103. [PubMed: 19596237]

8. Martinez P, Thanasoula M, Munoz P, et al. Increased telomere fragility and fusions resulting from TRF1 deficiency lead to degenerative pathologies and increased cancer in mice. Genes Dev 2009;23:2060-75. [PubMed: 19679647]

9. Crabbe L, Verdun RE, Haggblom CI, Karlseder J. Defective telomere lagging strand synthesis in cells lacking WRN helicase activity. Science 2004;306:1951-3. [PubMed: 15591207]

10. Sabourin M, Tuzon CT, Zakian VA. Telomerase and Tellp preferentially associate with short telomeres in S. cerevisiae. Mol Cell 2007;27:550-61. [PubMed: 17656141]

11. Abreu E, Aritonovska E, Reichenbach P, et al. TIN2-tethered TPP1 recruits human telomerase to telomeres in vivo. Mol Cell Biol 30:2971-82. [PubMed: 20404094]

12. Marion RM, Strati K, Li H, et al. Telomeres acquire embryonic stem cell characteristics in induced pluripotent stem cells. Cell Stem Cell 2009;4:141-54. [PubMed: 19200803]

13. Tejera AM, Stagno d'Alcontres M, Thanasoula M, et al. TPP1 is required for TERT recruitment, telomere elongation during nuclear reprogramming, and normal skin development in mice. Dev Cell 2010;18:775-89. [PubMed: 20493811]

14. Shore D, Bianchi A. Telomere length regulation: coupling DNA end processing to feedback regulation of telomerase. EMBO J 2009;28:2309-22. [PubMed: 19629031]

15. Zhao Y, Sfeir AJ, Zou Y, et al. Telomere extension occurs at most chromosome ends and is uncoupled from fill-in in human cancer cells. Cell 2009;138:463-75. [PubMed: 19665970]

16. Britt-Compton B, Capper R, Rowson J, Baird DM. Short telomeres are preferentially elongated by telomerase in human cells. FEBS Lett 2009;583:3076-80. [PubMed: 19716824]

17. Surovtseva YV, Churikov D, Boltz KA, et al. Conserved telomere maintenance component 1 interacts with STN1 and maintains chromosome ends in higher eukaryotes. Mol Cell 2009;36:207-18. [PubMed: 19854131]

18. Miyake Y, Nakamura M, Nabetani A, et al. RPA-like mammalian Ctc1-Stn1-Ten1 complex binds to single-stranded DNA and protects telomeres independently of the Pot1 pathway. Mol Cell 2009;36:193-206. [PubMed: 19854130]

19. Stewart SA, Hahn WC, O'Connor BF, et al. Telomerase contributes to tumorigenesis by a telomere length-independent mechanism. Proc Natl Acad Sci U S A 2002;99:12606-11. [PubMed: 12193655] 
20. Gonzalez-Suarez E, Samper E, Ramirez A, et al. Increased epidermal tumors and increased skin wound healing in transgenic mice overexpressing the catalytic subunit of telomerase, mTERT, in basal keratinocytes. Embo J 2001;20:2619-30. [PubMed: 11387197]

21. Artandi SE, Alson S, Tietze MK, et al. Constitutive telomerase expression promotes mammary carcinomas in aging mice. Proc Natl Acad Sci U S A 2002;99:8191-6. [PubMed: 12034875]

22. Bednarek AK, Chu Y, Slaga TJ, Aldaz CM. Telomerase and cell proliferation in mouse skin papillomas. Mol Carcinog 1997;20:329-31. [PubMed: 9433476]

23. Parkinson EK, Fitchett C, Cereser B. Dissecting the non-canonical functions of telomerase. Cytogenet Genome Res 2008;122:273-80. [PubMed: 19188696]

24. Maida Y, Yasukawa M, Furuuchi M, et al. An RNA-dependent RNA polymerase formed by TERT and the RMRP RNA. Nature 2009;461:230-5. [PubMed: 19701182]

25. Park JI, Venteicher AS, Hong JY, et al. Telomerase modulates Wnt signalling by association with target gene chromatin. Nature 2009;460:66-72. [PubMed: 19571879]

26. Hills M, Lansdorp PM. Short telomeres resulting from heritable mutations in the telomerase reverse transcriptase gene predispose for a variety of malignancies. Ann N Y Acad Sci 2009;1176:178-90. [PubMed: 19796246]

27. Davoli T, Denchi EL, de Lange T. Persistent telomere damage induces bypass of mitosis and tetraploidy. Cell 141:81-93. [PubMed: 20371347]

28. Henson JD, Cao Y, Huschtscha LI, et al. DNA C-circles are specific and quantifiable markers of alternative-lengthening-of-telomeres activity. Nat Biotechnol 2009;27:1181-5. [PubMed: 19935656] 